NOTORIEDAD DE

\section{LA COMUNICACIÓN}

INSTITUCIONAL A TRAVÉS

\section{DE LA DIPLOMACIA}

\section{Ricardo Ruiz de la Serna}

\section{Profesor}

Facultad de Humanidades y Ciencias de la Comunicación. Universidad San Pablo CEU. Paseo Juan XXIII, 6, Despacho 4, 28040 Madrid (España) - Tlfn.: (+34) 914564200 - Email: rrserna.fhmeceu.es

\section{Resumen}

El concepto moderno de Diplomacia pública ha ido unido al desarrollo de los medios de comunicación de masas, la sociedad del conocimiento y las nuevas formas de organización. Así, estamos asistiendo a un cambio en las técnicas de la Diplomacia que está tomando algunas de las prácticas tradicionales de la Comunicación Institucional unidas a los usos más recientes de los descubrimientos tecnológicos; en especial de la red 2.0. Este artículo describe algunas de estas técnicas y su uso en la Diplomacia pública.

\section{Palabras clave}

Comunicación institucional, diplomacia pública, imagen del Estado

Key Words

Corporate communications, public diplomacy, image of the State

\section{Abstract}

This article describes how Public Diplomacy is using some techniques of Corporate Communications and the development of a new concept of Public Diplomacy determined by the web 2.0. 


\section{Introducción}

Los estudios sobre Comunicación Institucional han centrado su atención tanto en el ámbito empresarial y corporativo como en el de la Administración Pública en sus distintos niveles: Estatal, Autonómico, Local, etc. (Canel: 2008). Sin embargo, la acción exterior del Estado -entendida como aquella tendente a afirmar la presencia del Estado en el exterior y la defensa de sus nacionales y sus intereses- ha gozado de menor atención en la literatura especializada.

No obstante, en los últimos años, diversos factores han modificado las formas tradicionales de la diplomacia y la forma de actuar de sus agentes. El desarrollo tecnológico ha alterado sustancialmente la forma que los Estados tenían de comunicarse con los distintos públicos. Por otra parte, la Comunidad Internacional ha presenciado la aparición de nuevos agentes estatales y el creciente protagonismo de las organizaciones internacionales. Estos cambios han ido unidos al desarrollo de nuevas formas de organización social a los que se ha ido adaptando la comunicación institucional. Así, las empresas y organizaciones privadas han sido pioneras en la explotación de los recursos que la Red 2.0 ofrece.

\section{Objetivos}

El objetivo de este artículo es describir en general qué técnicas de la Comunicación corporativa externa se están utilizando hoy en la Diplomacia pública así como las tendencias en lo relativo al empleo de los recursos que brinda la red 2.0.

\section{Metodología}

El método es descriptivo de la comunicación institucional de fuentes diplomáticas con una contribución de la bibliografía más reciente relacionada con los cambios so- ciales y su relación con las nuevas tecnologías. Se recurre a estadísticas elaboradas y publicadas por terceros. 


\section{De la diplomacia abierta a la diplomacia pública}

Históricamente, la Diplomacia es el campo de actividad de la acción exterior del Estado y, más recientemente, de las Organizaciones internacionales. Así, gracias a los cauces diplomáticos los Estados establecen relaciones y resuelven sus controversias.

Cuando termina la diplomacia, suele entenderse que comienza la guerra o, al menos, desaparece la buena relación entre los miembros de la Comunidad Internacional.

Sin embargo, también desde muy antiguo, la diplomacia - o más bien los diplomáticosestuvieron vinculados a las tareas de inteligencia y propaganda. Los Embajadores suministraban información a los príncipes sobre aquello que acontecía en los lugares en que estaban destinados y promovían la buena imagen de aquellos Estados a los que representaban. Esta promoción comprendía tanto los aspectos discursivos verbales -hablaban en nombre del Príncipecomo los simbólicos. Desde la vestimenta de los Embajadores hasta la lengua en que se dirigían a los soberanos extranjeros marcaban la imagen del Poder al que representaban.

A menudo, pues, la imagen del país a través de la diplomacia quedaba circunscrita a los soberanos extranjeros y, a lo sumo, a las poblaciones en que una Embajada estaba establecida. Sin duda, esto co- menzó a cambiar ya a partir del siglo XVII con al aparición de la diplomacia moderna. Así, por ejemplo, el uso de la arquitectura efímera, la fiesta barroca y la incipiente aparición de los medios de comunicación de masas contribuyeron a que los Embajadores pudiesen transmitir a los habitantes de un territorio - y no sólo a sus soberanosla imagen que el Monarca al que servían deseaba transmitir.

Así, desde entonces, la diplomacia ha ido ocupándose de la promoción de la imagen del Estado en el Exterior no sólo respecto de los otros Estados, sino más en general frente a la opinión pública, que va ganando creciente importancia en el siglo XIX y sobre todo en el siglo XX a la hora de hablar de un régimen de opinión.

La creciente importancia de la opinión pública en el diseño y ejecución de las políticas exigió que la diplomacia dejase de ser un cauce de la relación exclusiva con los Estados y pasase a ser, más bien, una forma de relacionarse con los distintos agentes políticos y sociales del exterior sin que los Estados agotasen esa definición.

De este modo, se irá consolidando el término de diplomacia pública. Aunque las primeras referencias al término ${ }^{\mathrm{i}}$ las encontramos en la oratoria parlamentaria, se 
referían a lo que llamaríamos diplomacia abierta frente a la diplomacia secreta:

My hon. and gallant friend is a man much enamoured of public diplomacy. He remembers, no doubt, that three weeks ago the Duc de Gramont went to the Legislative body of France and made an announcement as to the policy which the French Government would pursue with respect to Prussia. The result of that example of public diplomacy no doubt greatly encouraged my hon. And gallant friend. Then we have a specimen in the speech of my hon. And gallant friend of the kind of public diplomacy which we should have in this case if his hopes and desires were realized. ${ }^{\text {ii }}$

El concepto de lo que hoy conocemos como diplomacia pública se irá acuñando a lo largo del siglo $\mathrm{XX}^{\mathrm{iii}} \mathrm{y}$ terminará defi- niendo lo que hoy consideramos parte de la actividad natural de un diplomático.

Esta función de influencia que tiene hoy la Diplomacia pública la encontramos expuesta con claridad- unida a los fines más tradicionales de la Diplomacia- en la Carta de la Comisión Asesora de los Estados Unidos sobre Diplomacia pública-, cuya función se describe en el apartado I, que reza: "The United States Advisory Commission on Public Diplomacy appraises U.S. Government activities intended to understand, inform, and influence foreign publics". iv

Para esta actividad de comprensión, información e influencia, la diplomacia pública se ha venido sirviendo de las técnicas de comunicación institucional desarrolladas en el seno de organizaciones privadas.

\section{El uso de las técnicas de comunicación institucional para los fines de la Diplomacia pública}

Las técnicas propias de la comunicación institucional sirven a la Diplomacia pública para la construcción de la imagen del Estado. En este sentido, los Ministerios y las Embajadas se dotan de Gabinetes de prensa, de portavoces y de asesores de comunicación. No es tan frecuente la creación de gabinetes de comunicación en el sentido técnico de la Comunicación Institucional (Martín Martín 2006)
Estos equipos despliegan la actividad habitual que en las empresas realizan los departamentos de comunicación en lo que se refiere a la comunicación externa (Rius Mengotti 2007 y Martín Martín 2006)).

Así, las Embajadas suelen tener organizado un departamento de relaciones con la prensa al frente del cual está normalmente un Jefe o Agregado de Prensa. 
Independientemente del nombre que reciba, será el encargado de dirigir las relaciones con la prensa, para lo cual debe realizar fundamentalmente las siguientes actividades:

1. Hacer un listado de medios de comunicación y un riguroso seguimiento tanto de los medios de comunicación existentes como de los periodistas que trabajan en ellos y los cambios de trabajo de estos últimos.

2. Establecer nuevos contactos entre la prensa y mantener la relación con ellos, estando a su disposición cuando necesiten información.

3. Proporcionar noticias a los medios de comunicación.

4. Hacer un seguimiento y análisis de los medios de comunicación.

En realidad, estas actividades no son muy diferentes de las que realiza un gabinete de prensa del sector privado.

\subsection{Listado de prensa $y$ seguimiento de los cambios que se produzcan}

Una de las primeras tareas del Departamento de Prensa es elaborar un listado de medios de comunicación y de los periodistas que en ellos trabajan. Además, habrá que clasificar los medios, pudiendo distinguir en primer lugar, entre prensa, radio y televisión. A partir de ahí, podrán hacerse otras clasificaciones en función de diversos criterios: por ejemplo, si es un medio de comunicación general o sectorial y/o especializado; ámbito de información (económico, político, social, etc.); si es un medio favorable al interés de la Embajada o no; si es prensa escrita o digital; la periodicidad de la publicación, etc.

En todo caso, será muy importante hacer un seguimiento continuo de en qué medios trabajan los periodistas en cada momento, para que el trato con el periodista sea lo más personalizado posible. De esta manera, es posible gestionar con precisión la información que se le suministra y la repercusión que pueda tener.

En el listado, han de incluirse todos los datos de contacto del medio de comunicación y de los periodistas que en él trabajan así como la sección a la que pertenecen. Asimismo, es habitual incluir la fecha de actualización del listado.

\subsection{Establecer} nuevos contactos entre la prensa $y$

\section{mantener la relación con ellos suministrándoles información}

Las formas de presentarse y acercarse al periodista son las habituales de la comunicación Institucional. Se envían comunicados a los medios anunciando el nombramiento del nuevo Jefe o Agregado de Prensa dando sus datos de contacto. También se recurre a notas de prensa con noticias o 
informaciones relevantes y se establece después el contacto personal para ampliarlas. Del mismo modo, se suelen organizar reuniones y seminarios de trabajo aprovechando la visita de alguna personalidad o la ocurrencia de algún hecho de interés informativo. A veces, se patrocinan Jornadas, Seminarios o Cursos en los que la Embajada colabora para hacer un primer contacto con los periodistas y retomarlo después.

\subsection{Proporcionar noticias a los medios de comunicación}

Las notas de prensa o comunicados son el medio más habitual de hacer llegar una noticia a los medios de comunicación. No son habituales los comunicados de prensa audiovisuales. Las notas se suelen enviar a aquellos medios o periodistas incluidos en los listados del Departamento de Prensa. Estas notas de prensa suelen ir acompañadas de elementos que ayudan a comprender la noticia en toda su amplitud o que la hacen más atractiva para los medios, tales como fotografías, estadísticas, gráficos, mapas, etc.

Al igual que en el sector privado, suele prestarse mucha atención a los aspectos formales de las comunicaciones. Habitualmente se remiten impresas en papel -o por correo electrónico- con logotipo oficial, por una sola cara, a doble espacio o con unos márgenes adecuados, sin subrayados para que sea el periodista el que resalte lo que le interese $y$, finalmente, con los datos de contacto de la persona de la Embajada a la que podrá dirigirse el periodista para ampliar la información.

Las conferencias de prensa se utilizan con menor frecuencia y sólo cuando la importancia de la noticia así lo requiere. Los preparativos de la conferencia son los habituales en el sector privado, especialmente la elaboración de listados de posibles preguntas y repuestas.

También es frecuente la elaboración de un dossier de prensa que se suele repartir a los periodistas que acuden a los eventos informativos o que se envía con posterioridad a aquellos periodistas que hubieran confirmado su asistencia pero que, al final, no hayan estado presentes.

Los eventos informativos -al igual que sucede en el sector privado- han de anunciarse con la suficiente antelación y la fecha y hora debe ser cuidadosamente escogida para poderla integrar en el ciclo de noticias (evitar que coincida con otros eventos importantes, intentar no hacerla en lunes ni a primera hora de la mañana).

Otra forma de dar información a los medios es la entrevista. En este caso, el Jefe de Prensa suele ser el receptor de la solicitud del periodista de entrevistar a algún cargo de la Embajada y será él quien deba ajustar la agenda de periodista y entrevistado para hacerlo posible. Además, estará presente en la entrevista para presentar a ambos y hacer de anfitrión. 


\subsection{Seguimiento de los medios de comunicación}

El Jefe de Prensa suele hacer un seguimiento diario de las informaciones publicadas en la prensa con el objetivo de hacer una valoración sobre la eficacia del departamento de relaciones con la prensa e, incluso, de solicitar a los medios la opor- tuna rectificación en caso de que se haya publicado o emitido información incorrecta.

Para ello, se elabora un dossier con los recortes de prensa que se van recopilando, lo que le facilitará al Jefe o Agregado de Prensa la tarea de evaluar su propio trabajo, así como de exponer dichos resultados a sus superiores.

\section{El uso de la red 2.0 en la diplomacia pública}

A las técnicas ya señaladas, se une un creciente uso de los recursos que brinda Internet, especialmente de los propios de la red 2.0 .

Por ejemplo, la Subsecretaria de Diplomacia y Asuntos Públicos Judith A. MC Hale declaraba ante el Congreso de los Estados Unidos en el trámite de su ratificación:

[...] new technology, used effectively and creatively, can be a game changer. Communications advances provide unprecedented opportunities to engage people directly, to connect them to one another, and to dramatically scale up many traditional public diplomacy efforts. They provide us the opportunity to move from an old paradigm in which our government speaks as one to many, to a new model of engaging interactively and collaboratively across lines that might otherwise divide us from people around the world. We must create an institutional framework that can take full ad- vantage of new media, with an understanding that these tools must be carefully tailored to particular circumstances and always used in the service of a larger strategy.

Así, los recursos tecnológicos - a diferencia de las técnicas más tradicionales de comunicación institucional- se configuran como recursos estratégicos y no sólo tácticos en el proceso comunicativo. Se relacionan con el modo de comprender el mundo y su organización y no sólo con los medios de transmisión de un mensaje.

En este sentido, la propia McHale señalaba el uso que se había hecho tanto de Youtube como de otros recursos tecnológicos desde el propio Departamento de Estado:

Early forays into social networking and new media show real potential. Working with a wide range of private and NGO partners, the State Department last year launched the Democracy Video Challenge, a global online contest that asked citizens to create and post 
on YouTube short videos expressing their perspectives on democracy. More than 900 people from 95 countries submitted videos, including more than 50 from Iran. Over 70 of our embassies joined the effort, hosting screenings and encouraging local participation. While the numbers remain modest, the early success of the Democracy Video Challenge suggests the power of new communications tools to connect people around the world and foster global dialogues that are truly interactive.

En este proyecto, se apostó no sólo por la transmisión de mensajes -algo propio de la Diplomacia pública desde tiempos de $\mathrm{Mu}$ rrows- sino además por la interacción a la hora de elaborar las propias políticas. De este modo, las Embajadas estadounidenses se convirtieron no sólo en emisoras de mensajes, sino también en receptoras de éstos de forma organizada. Conviene advertir que esta decisión forma parte de un programa político más ambicioso tendente al compromiso de escuchar -en palabras del Presidente Barack Obama- los pensamientos e ideas de los demás. Así lo señalaba, en el discurso inaugural del programa Democracy Video Challenge la propia Subsecretaria de Estado:

President Obama and Secretary Clinton have both underscored the importance of democracy as well as engaging with others to listen to their thoughts and ideas. The Democracy Video Challenge helps accomplish both of these goals. The Challenge asks people around the world to create a short video that completes the phrase, "Democracy is...." It also provides a platform for individual voices to be heard worldwide. We in the State Department and our partners have designed the Challenge to provide people around the world with the opportunity to express their views and to define democracy in their own terms.

Así, el uso de los medios de la Diplomacia pública no sólo para influir sino también para escuchar y, en ese sentido, entender la realidad de los países en que la acción exterior del Estado se despliega resulta novedosa e interesante.

Ha habidos críticas al propio uso de las redes con fines políticos precisamente por la carga ideológica que su propio diseño y sus aplicaciones - por ejemplo ciertos buscadores- llevarían aparejados así como por su falta de legitimidad democrática (Cassin 2008) .

Las voces críticas respecto a esta tendencia en el uso de las redes sociales para los fines de la diplomacia pública también destacan sus limitaciones. Mozorov duda de que la burocracia americana with its infinite levels of communication and gatekeeping would be able to come up with "an institutional framework" for the anti-institutional platforms they seek to embrace. A esto habría que sumarle el poder generador de ruido y rumores que tiene la propia red, un potencial que puede utilizarse para los fines propios de la comunicación persuasiva (Rosen 2000). 
He aquí una de las limitaciones que la diplomacia pública debe afrontar: las redes sociales -y en general el propio funcionamiento de la Red 2.0- es difícilmente compatible con las organizaciones jerarquizadas y centralizadas. El éxito de la Red en esta nueva etapa radica, precisamente, en su adaptación a las nuevas formas de las organizaciones, es decir, la red y la descentralización (Giddens 2006) El proceso inmediato de los datos en cualquier lugar ha permitido que las organizaciones se rediseñen, se descentralicen y se vuelvan flexibles y menos burocráticas. Como anticipaba Castells (Castells 2005) estamos ante dimensiones diferentes de un proceso fundamental: la burocracia tradicional se está desintegrando.

A esto se añade que la Red 2.0 y las nuevas formas de organización social suponen un cambio en las relaciones entre emoción, cognición y política (Castells: 2009). Las redes sociales desempeñan un papel importante en la definición del comportamiento político y

esta tendencia irá en aumento. Dada la predisposición de las personas a agruparse en torno a quienes comparten sus ideas, las redes fomentarán la participación política. Emociones como el miedo o la esperanza se convierten en determinantes en el proceso político (Castells: 2009) y sobre ellas ha de influir la Diplomacia pública a la hora de cambiar la imagen exterior de los Estados.

Así, en cuanto tendencia -con todas las limitaciones que se quiera- es claro que la diplomacia pública debe integrar los mecanismos tecnológicos del mismo modo que tuvo que adoptar técnicas y métodos más tradicionales de la Comunicación Institucional. Siguiendo a Micah Sifry y Andrew Rasej, la actividad política en nuestro siglo trata de cómo los ciudadanos se relacionan más entre sí y con los gobiernos. De este modo, la diplomacia pública puede aprovechar los cauces que le brinda la tecnología para transmitir su mensaje a públicos que quedan fuera de los medios más tradicionales de la Comunicación institucional, por ejemplo los jóvenes. A propósito de este público juvenil, Boschma (Boschma 2008) señala la ineficacia de las campañas publicitarias tradicionales en televisión, radio y prensa y la advertencia de Fallows sobre la creciente desconfianza de los ciudadanos hacia los medios más tradicionales (Fallows 1997) sigue vigente. Por fin, el propio funcionamiento de la red genera nuevas formas de relación entrelos ciudadanos $y$ los distintos operadores en la red ajenos a los modos tradicionales de interacción (Tapscott y Williams 2007; Jenkins 2009)) Podríamos extender las conclusiones de ambos a las tareas de diplomacia pública si analizamos, por ejemplo, la escasa incidencia que las campañas tradicionales organizadas por el Broadcasting Board of Governors en los mismos medios han venido teniendo.

Para analizar este punto podemos estudiar los sucesivos indicadores de la imagen de los Estados Unidos que publica el Pew 
Research Center. Tomemos el más reciente AMERICA'S PLACE IN THE WORLD 2009. An Investigation of Public and Leadership Opinion About International Affaire. ${ }^{\text {vi }}$ A pesar de todos los esfuerzos de difusión realizados por los diversos medios dependientes del Broadcasting Board of Governors, China aparece como una potencia creciente y poderosa y los Estados Unidos como una potencia debilitada.

De este modo, sí cabe concluir que los recursos que brinda la Red 2.0, con limitaciones pero también con gran potencial, serán imprescindibles para las labores de diplomacia pública en el futuro. Al igual que sucedió con las técnicas más tradicionales de la Comunicación institucional, la diplomacia tendrá que servirse de las redes sociales, los blogs corporativos y las demás herramientas que brinda el desarrollo tecnológico para la construcción de la imagen exterior de los Estados.

Siguiendo a Noya, es interesante señalar cómo en el caso de España, se ha optado por la superación del concepto de marcapaís en aras del de imagen exterior de España. ${ }^{\text {viii }}$

De este modo, sí cabe concluir que los recursos que brinda la Red 2.0, con limitaciones pero también con gran potencial, serán imprescindibles para las labores de diplomacia pública en el futuro. Al igual que sucedió con las técnicas más tradicionales de la Comunicación institucional, la diplomacia tendrá que servirse de las redes sociales, los blogs corporativos y las demás herramientas que brinda el desarrollo tecnológico para la construcción de la imagen exterior de los Estados.

Siguiendo a Noya, es interesante señalar cómo en el caso de España, se ha optado por la superación del concepto de marcapaís en aras del de imagen exterior de España8

Así lo ha valorado la propia Diplomacia española. El Presidente del Gobierno José Luis Rodríguez Zapatero establecía este objetivo en su Discurso ante los participantes en la V Conferencia de Embajadores de España:

El tema de la reunión de este año de la Conferencia era el de la diplomacia pública, es decir, una nueva dimensión internacional de nuestra política exterior, a la que otorgo gran relevancia. Esa diplomacia pública tiene un objetivo: fortalecer la imagen y la percepción de nuestro país, dar a conocer a todas las opiniones públicas nuestra realidad y nuestras políticas, nuestros principios comunes como país en este tiempo. Contamos ya con algunos instrumentos de gran valía: el Instituto Cervantes y las Casas América, Asia, África y Árabe. Debemos mejorar nuestra capacidad de acción. ${ }^{\text {ix }}$

El Presidente encomendaba esta tarea a una comisión en que se integrarían sectores que, hasta el momento, habían estado más alejados de las funciones de diplomacia pública: la sociedad civil, los medios de comunicación, el mundo de la cultura y la empresa: 
Una Comisión de Diplomacia Pública se va a encargar de esta tarea y va a incorporar a miembros del Gobierno, de la sociedad civil, de los medios de comunicación, del mundo de la cultura y de la empresa, para analizar cada año nuestra proyección exterior en todos los ámbitos y proponer futuras líneas de actuación.

Como se ha visto más arriba, el Presidente ha centrado los esfuerzos de diplomacia pública en el Instituto Cervantes y las $\mathrm{Ca}$ sas, y son precisamente estas instituciones las que han desarrollados sus sitios web dotándolos de los recursos que brinda la Red 2.0.

Los esfuerzos ministeriales siguen la tendencia de ampliar la comunicación a ámbitos que históricamente quedaron fuera de la acción estrictamente diplomática. En realidad, esta función de diplomacia pública ni siquiera se ha encomendado en exclusiva a órganos tradicionales de la diplomacia, sino que se han creado otras instituciones complementarias que forman la red de diplomacia pública. Así lo declaraba el Ministro de Asuntos Exteriores Miguel Ángel Moratinos en su discurso inaugural de la V Conferencia de Embajadores de España ${ }^{\mathrm{x}}$ :

El Gobierno español ha realizado una apuesta a largo plazo y potenciará su red de diplomacia pública integrada básicamente por el Instituto Cervantes para la promoción del español y la cultura iberoamericana, con 77 centros en 41 países, las Casas, la $A E$ CID o la Fundación ONUART.

La red de Casas la integran consorcios públicos en los que intervienen todos los niveles de la Administración (estatal, autonómico y local), así como la sociedad civil. A lo largo del último mandato se han creado tres nuevos equipamientos públicos: Casa Árabe, Casa África y Casa Sefarad-Israel, que se han unido a las ya existentes Casa América y $\mathrm{Ca}$ sa Asia, y a las que pronto se sumarán Casa del Mediterráneo y Casa España-Estados Unidos.

Es precisamente en los sitios web de las Casas y del Instituto Cervantes donde vamos a encontrar el mayor despliegue de los medios que la Red 2.0 ofrece para la comunicación institucional de fuentes.

\section{Conclusiones}

La diplomacia ha dejado de ser sólo el modo que los Estados tienen de relacionarse entre sí y se ha convertido en una forma de influir en la construcción de la imagen exterior del Estado al servicio de su política.
Los cambios sociales y organizativos -y en especial el auge de la descentralización y la organización en red- han ido parejos al desarrollo tecnológico que supone Internet 2.0. Así, a los recursos tradicionales de la comunicación institucional -que se man- 
tienen- los Estados tratan de incorporar los medios que el avance tecnológico brinda para influir en el exterior.

La tendencia, pues, es el aumento de los recursos y medios destinados a la construc- ción de la imagen exterior del Estado a través de medios tecnológicos que complementarán las prácticas ya tradicionales de la comunicación institucional.

\section{Referencias}

BASSIN, B. (2008) Googléame. La segunda misión de los Estados Unidos. México D.F., EDit. Fondo de Cultura Económica.

BOSCHMA, J (2008) Generación Einstein. Más listos, más rápidos y más sociables. Madrid, Edit. Gestión 2000.

CANEL, Ma José. (2007) Comunicación de las Instituciones públicas, Madrid, Edit. Tecnos.

CASTELLS, M (2005) La sociedad red. Madrid, Edit. Alianza.

CASTELLS, M (2009) Comunicación y poder. Madrid, Edit. Alianza.

FALLOWS, J (1997) Breaking The News. How The Media Undermine American Democracy. New York, Edit. Vintage Books.

GIDDENS, A (2006) Sociología. Madrid, Edit. Alianza.

JENKINS, H (2009) Fans, blogueros y videojuegos, Barcelona, Edit. Paidós.

MARTÍN MARTÍN, F. (2006) Comunicación Empresarial e Institucional. Madrid, Edit. Universitas y Asociación APM. (4 ${ }^{\mathrm{a}}$ Edición)
MOZOROV, E. The Future of Public Diplomacy 2.0, en http: / / neteffect.foreignpolicy.com/posts/ 2009/06/09/the future of public diplomacy 20

NOYA, J. La imagen exterior de España: nuevos escenarios y viejos problemas, en http:/ / www.real institutoelcano.org/wps/portal/rielcano/contenido ?WCM GLOBAL CONTEXT $=/$ elcano/elcano es /zonas es/imagen+de+espana/dt60-2009

RIUS MENGOTTI, M, (2007) Relaciones públicas con los medios de comunicación: perspectiva empresarial en BARQUERO CABRERO, J.D. y BARQUERO CABRERO, M. Manual de las Relaciones Públicas, Comunicación y Publicidad.

ROSEN, E (2000) The Anatomy of Buzz. New York, Edit. Currency Book

SIFRY, M. Y RASEY, A. The Rise of e-Diplomacy, en http: / / www.politico.com/news/stories/0609/233 $\underline{10 . h t m l}$

TAPSCOTT D. y WILLIAMS A.D. (2007) Wikinomics. La nueva economía de las multitudes inteligentes. Barcelona, Edit. Paidós

\section{Cita de este artículo}

RUIZ DE LA SERNA, R. (2010) Notoriedad de la comunicación institucional a través de la diplomacia. Revista Icono14 [en línea] 1 de Julio de 2010, Año 8, Vol. 2. pp. 291-303. Recuperado (Fecha de acceso), de http://www.icono14.net 


\section{NOTAS}

${ }^{\text {i } E x i s t e n ~ r e f e r e n c i a s ~ d e ~} 1869$ y 1870 en la prensa, pero se omiten aquí por no tratarse de órganos del Estado.

ii Gladstone, W.E.; Discurso de los días 8 y 10 de agosto de 1870 relativo a la neutralidad de Bélgica. Accesible en http://www.gutenberg.org/files/10990/10990-8.txt

iii Es probable que las primeras aclaraciones conceptuales naciesen en el seno de la Unites States Information Agency como alternativa al uso del término propaganda, que estaba muy desprestigiado, y como parte de la redefinición de la política exterior estadounidense. Dizard (2004).

${ }^{\text {iv }}$ http://www.state.gov/r/adcompd/charter/104510.htm

${ }^{\mathrm{v}} \mathrm{http} / / /$ www.state.gov/r/remarks/124155.htm

${ }^{v i}$ http://www.state.gov/r/remarks/129637.htm

vii http://people-press.org/reports/pdf/569.pdf

viii http://www.realinstitutoelcano.org/wps/portal/rielcano/contenido?WCM GLOBAL CONTEXT=/ elcano/elcano es/zonas es/imagen+de+espana/dt60-2009

${ }^{i x}$ http://www.la-moncloa.es/Presidente/Intervenciones/Discursos/prdi20080908.htm

${ }^{\mathrm{x}}$ http://www.maec.es/es/MenuPpal/Actualidad/Declaracionesydiscursos/Paginas/discursoministro 20080908.aspx 